\title{
A Dynamic High-Order Equivalent Modeling of Lithium-Ion Batteries for the State-of-Charge Prediction Based on Reduced- Order Extended Kalman Filtering Algorithm
}

\author{
Emmanuel Appiah, Shunli Wang, Chuanyun Zou, Hongying Zhang, \\ Etse Dablu Bobobee, Manasah Musariri, Islam Md Monirul \\ School of Information Engineering, Southwest University of Science and Technology, \\ Mianyang 621010, China
}

The work is supported by the National Natural Science Foundation of China (No. 61801407), Sichuan Science and Technology program (No. 2019YFG0427), China Scholarship Council (No. 201908515099), and Fund of Robot Technology Used for Special Environment Key Laboratory of Sichuan Province (No. 18kftk03).

\section{Abstract}

Detection of battery power has always been the core of the battery management system of electric vehicles, and the fast and accurate estimation of charged state can guarantee the safe operation of electric vehicles. The key to improving accurate state-of-charge estimation is an appropriate model establishment coupled with a suitable estimation algorithm. This research seeks to adopt and accomplish a lithium-ion battery state-of-charge estimation based on the Gaussian function to build up the open-circuit voltage algorithm. A reduced-order extended Kalman filtering algorithm is proposed with hybrid pulse power characterization parameter identification to estimate the battery characterization state-of-charge. The model's parameters in different state-of-charge points are calculated through the lithium-ion battery's charge and discharge process; the 2RC modeling correction method and Reducedorder extended Kalman filter method are used separately based on the High-order equivalent 2RC modeling. The Experimental results show that the above method can achieve state-of-charge estimation more accurately and conveniently, providing a certain reference value for the rational management and distribution of power lithiumion batteries. The maximum error of state-of-charge estimation based on the established high-order equivalent 2RC model using the Reduced-order extended Kalman filtering algorithm is less than $1.85 \%$. The REKF algorithm achieved a maximum voltage error of $0.0409 \mathrm{~V}$ and an average error of $0.0299 \mathrm{~V}$ and therefore can satisfy the accuracy of the battery management system application needs.

Keywords: Lithium-ion battery; state-of-charge; high-order equivalent 2RC modeling; open-circuit voltage; parameter identification; reduced-order extended Kalman filtering algorithm

DOI: $10.7176 /$ JETP/11-3-03

Publication date:June $30^{\text {th }} 2021$

\section{Introduction}

As the technology in today's society becomes increasingly mobile and as information networks grow, the importance of lithium-ion batteries (LIBs) increases considerably with its use in most electronic equipment and many other applications because of a range of advantages, such as a high operating voltage, small self-discharge, and no memory effects. The state-of-charge (SOC) estimation of the lithium-ion battery (LIB) and battery pack is a core technology in the battery management system (BMS) of electric vehicles (EV). Accurate SOC estimation can effectively enhance the safety and energy efficiency of batteries [1,2]. Recently, amidst the increasing concern for the environment and energy power generation challenges, many attempts have been made to install LIBs in plug-in hybrid vehicles or electric vehicles but the biggest obstacle comforting LIBs is the issue of safety. This affects the utilization efficiency of its capacity and life directly, leading to accidents in several cases for future automotive energy power systems, and has become a high strategic priority $[3,4]$. For the lithium-ion battery to be safe and reliable, and operation needs to be monitored and managed by the battery management system there needs to be enhancement of safety performance and improvement of consistency, and elimination of explosion risk [5]. The accuracy of estimating the SOC of the battery is one of the important criteria reflecting the quality of the battery management system which is necessary for the power application of the LIB to establish a solid position in the future such as being used as the power sources in electronic vehicles. With this in mind, [6, 7] suggest that further improvements will be needed concerning the output characteristic, cycle characteristic, and safety of the battery in the future and avoid abuse, irrational use and ensure safety and extend the life of batteries. Accurate SOC estimation is an insurmountable concern due to the immature management of the associated BMS equipment [8]. The BMS is used for energy supply with good stability, efficiency, and reliability requirements aiming to avoid safety issues by monitoring battery status and optimizing usage [9]. A lithium-ion battery is a complex non-linear system, with a complex working condition and sensor measurement process which produces an inevitable noise, that makes accurate SOC estimation extremely difficult [10-12]. Sequentially, to improve the performance of LIBs, many researchers have studied the high efficiency of SOC estimation of the LIB and there are several methods 
proposed to estimate the SOC. Existing SOC estimation methods are mainly divided into the following categories [13], safety time method [14], internal resistance method, a practical electrical power source which is a linear electric circuit [15], and Kalman filter method, observed over time containing statistical noise and other inaccuracies [16].

In the last two decades, lithium-ion battery technology has made incredible advances in the world and the industry has globally reached about $\$ 15.5$ to $\$ 16$ billion [17]. Its application range has penetrated various industries, such as new energy vehicles, portable devices, aviation, and household appliances. In the accurate estimation process of the SOC of the lithium-ion battery, the construction of the battery equivalent model characterizes the dynamics of the battery and occupies an important position [18]. The most common battery structure in current applications includes electrochemical models, neural network models, and equivalent circuit models [19, 20]. A comparative study on global optimization methods for parameter identification of different equivalent circuit models of lithium-ion batteries is discussed in [21] and the state-of-charge estimation of lithium-ion batteries based on the novel reduced order electrochemical model presented in [22]. A study of a new fractional-order model for estimating the state-of-charge of lithium-ion batteries and a study of a dynamic linear model of the lithium-ion battery state-of-charge estimation method based on a simplified model is proposed in [23]. The equivalent circuit model of lithium-ion batteries is enhanced in [24] and improved package equivalent circuit modeling method in [25]. The equivalent circuit model shows the complex dynamic characteristics of the battery during use, specifically the dynamic response in the circuit loop. The use of circuit knowledge and calculus to establish circuit equations to study the battery characteristics, and the resulting convenience has been widely used in the engineering field. A study on the open-circuit voltage and state-of-charge characterization of high capacity lithiumion batteries under different temperatures is undertaken by [26] and an improved adaptive estimator for state-ofcharge estimation of lithium-ion batteries is carried out in [27]. In [28] the real-time study of the disequilibrium transfer in vanadium flow batteries at different states-of-charge via refractive Index detection is well studied.

The discharge test method uses a single discharge to release the continuous constant current of the battery until the cut-off voltage. The product of the discharge time and the discharge current can directly calculate the SOC value [29]. [30] examines incremental capacity analysis and differential voltage analysis-based state-ofcharge and capacity estimation for lithium-ion batteries. The State-of-charge inconsistency estimation of the lithium-ion battery pack using the mean-difference model and extended Kalman filter is covered by [31] and investigating the error sources of the online state-of-charge estimation methods for lithium-ion batteries in electric vehicles is also addressed in [5]. The current integration method estimates the battery SOC by accumulating the charge or discharge when the battery is charged and discharged, and also corrects the SOC value by the chargedischarge rate, and the battery temperature. At this present time and age, the commonly used methods in the estimation of lithium-ion battery SOC are the open-circuit-voltage (OCV) method, Ampere-hour(Ah) integral method, neural network method, and Kalman filter(KF) method [32]. These methods cannot only accurately estimate the SOC value, but are simple, easy to understand the principle used, and can also realize real-time estimation. The long-term integration process will accumulate effects, and the error will continue to expand, causing the SOC to deviate from the true value during the estimation process [33]. [34, 35] discussed an overview of model-based online state-of-charge estimation using Kalman filter family for lithium-ion batteries and selfadaptive filter for online state-of-charge estimation of lithium-ion batteries. The Kalman filter algorithm is combined with the open-circuit voltage method to find OCV, fitting the curve relationship between OCV and SOC, following the "prediction-actual measurement-correction" method, according to the minimum variance through continuous iterative operation $[36,37]$. [38, 39] examines an online internal resistance measurement application in lithium-ion battery capacity and SOC estimation [40,41] used parameter identification and extended Kalman filtering for SOC estimation and adaptive extended Kalman filtering to estimate SOC effect. [42] used fractional order extended Kalman filter to enhance the state-of-charge performance and estimate the lithium-ion batteries. [43] proposed a method for estimating the state-of-charge of lithium-ion batteries based on current-free detection algorithm. The work presented in [44] an online approach is introduced for SOC estimation and parameter updating using an improved adaptive cubature Kalman filter

Over time, the limitations of the Kalman filter algorithm are also found and therefore, an extended Kalman filter algorithm (EKF) is proposed. The extended Kalman filter algorithm is characterized by SOC prediction and the value expands the nonlinear function of OCV and SOC into a Taylor series and omits the second-order RC model or higher term so that an approximate linearization model can be obtained. In [45] the depicted Kalman filter method establishes and estimates battery SOC based on the extended Kalman filter that can reduce the estimation error. When the filtering error and the prediction error are small, the extended Kalman filter algorithm (EKF) is used to realize the SOC estimation in real-time and accurately. To extend the Kalman filter to the nonlinear systems, various modified KF algorithms have been proposed. The extended Kalman filter (EKF) algorithm employs the first-order Taylor series expansion to linearize the nonlinear system and the high-order terms are neglected in the linearization process, which will inevitably lead to large linearization errors [46, 47].

The utmost focal point of this research paper is the application of the Reduced-order EKF algorithm for SOC 
estimation. To reduce the linear system error and compute load of the KF, the Reduced-order extended Kalman filter (REKF) is adopted to SOC estimation based on a high-order 2RC equivalent model. The REKF algorithm utilizes the extension of the classic Kalman filter for a non-linear system where non-linearity is approximated using the first or second-order derivative, and its accuracy is higher than that of the KF algorithm. Hybrid Pulse Power Characterization (HPPC) is utilized to identify the model parameters and because of the strong coupling relationship between the operation conditions model parameters, the HPPC test can accurately describe the internal characteristics of the battery. As a result, the HPPC-REKF algorithm is proposed for SOC estimation. When the filtering error and the prediction error are small, the Reduced-order extended Kalman filter algorithm (EKF) is used to realize the SOC estimation in real-time and accurately. The divergence of REKF is primarily due to the linearization error for ignoring high-order terms, and the accuracy of REKF based SOC estimation is sensitive to the precision of the battery model. The rest of the paper is constructed as follows: The mathematical and theoretical analysis is conducted in section 2 including the high-order 2RC equivalent modeling, state-space iteration, opencircuit voltage test, and the proposed HPPC-EKF algorithm for SOC estimation. In section 3, the experiments are illustrated as well as their estimation effect results. Conclusions and future works are finally reported in Section 4.

Table 1. List of symbols

\begin{tabular}{cccl}
\hline Symbols & Description & Symbols & \multicolumn{1}{c}{ Description } \\
\hline $\boldsymbol{U}$ & Voltage & $\boldsymbol{w}_{\boldsymbol{k}}$ & The process noise of the system \\
$\boldsymbol{I}$ & Current & $\boldsymbol{v}_{\boldsymbol{k}}$ & The observation noise of the system \\
$\boldsymbol{R}$ & Internal ohmic resistance & $\boldsymbol{Q}_{\boldsymbol{k}}$ & The covariance of the process noise \\
$\boldsymbol{Q}$ & Battery capacity & $\boldsymbol{R}_{\boldsymbol{k}}$ & The covariance of the process observed noise \\
$\boldsymbol{y}$ & Output & $\boldsymbol{q}_{\boldsymbol{k}}$ & The mean of $w_{k}$ \\
$\boldsymbol{e}$ & Error & $\boldsymbol{r}_{\boldsymbol{k}}$ & The mean of $v_{k}$ \\
$\boldsymbol{x}$ & Input & $\lambda$ & Forgetting factor \\
\hline
\end{tabular}

\section{Mathematical Analysis}

\subsection{High-order equivalent 2RC modeling}

The accurate estimation of the SOC of a battery is one of the most important issues of an electrical vehicle's BMS, and the foundation of precise SOC estimation is an accurate battery model. The first-order RC model which is the simplest model is composed of a resistance-capacitance circuit in series with ohm internal resistance and is extensively used in battery SOC estimation [48]. The high-order 2RC equivalent model is utilized in this paper because it provides an accurate approximation for the lithium-ion battery dynamics model structure. This model is based on an equivalent circuit model whose parameters will be scheduled on the state-of-charge, temperature, and current direction as shown in Figure 1.

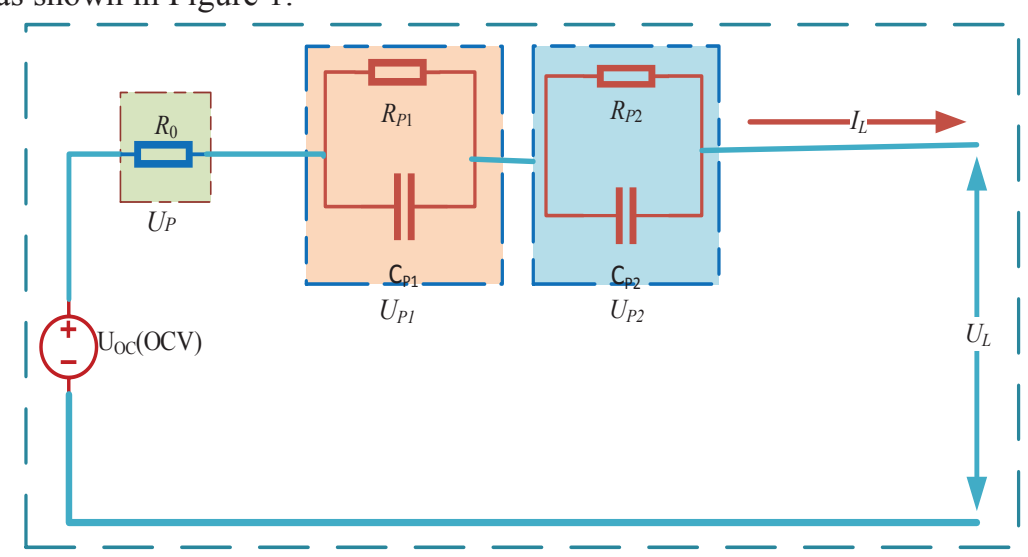

Figure 1. High-order equivalent (2RC) modeling

The lithium-ion battery models mainly include the equivalent circuit model, electrochemical model, empirical model, and mathematical model. The High-order equivalent model with two RC is an ideal battery model, which considers not only the static characteristics of the battery but also the transient characteristics. As shown in Figure 1, the battery model consists of the following five parts: (1) A voltage source $U_{O C}(\mathrm{OCV})$, utilized to describe the nonlinear monotonous relationship between OCV and SOC, which can be denoted as $U_{O C}(S O C)$; (2) $R_{0}$ is the ohmic resistance of the lithium-ion battery, which represents the ohmic polarization; (3) $R_{P 1}$ and $C_{P 1}$ are concentration polarization resistance and concentration polarization capacitance respectively, which describe the long-term polarization response; (4) $R_{P 2}$ and $C_{P 2}$ are electrochemical polarization resistance and electrochemical polarization capacitance respectively, which describe the short time polarization response; (5) $I_{L}$ and $U_{L}$ are the load current and terminal voltage, respectively. According to Kirchhoff's voltage law (KVL) and Kirchhoff's 
current law (KCL), the battery dynamic equation can be derived as expressed in Equation (1).

$$
\left\{\begin{array}{l}
U_{P}=-U_{P 1} \frac{R_{P 1}}{C_{P 1}}+\frac{I_{L}}{C_{P 1}} \\
U_{P}=-U_{P 2} \frac{R_{P 2}}{C_{P 2}}+\frac{I_{L}}{C_{P 2}} \\
U_{L}=U_{o c}(S O C)-U_{P 1}-U_{P 2}-I_{L} R_{o}
\end{array}\right.
$$

Among them, the open-circuit voltage can be characterized by the state variable SOC, and a nonlinear function relationship can be obtained. Using the knowledge of modern control theory, the equivalent circuit model is discretized and the definition of SOC is shown in Equation (2).

$$
\left\{\begin{array}{l}
I_{L}=\frac{U_{P 1}}{R_{P 1}}+C_{P 1}=\frac{d U_{P 1}}{d_{t}} \\
I_{L}=\frac{U_{P 2}}{R_{P 2}}+C_{P 2}=\frac{d U_{P 2}}{d_{t}} \\
U_{L}=U_{o c}(S O C)-U_{P 1}-U_{P 2}-I_{L} R_{o}
\end{array}\right.
$$

Where $R_{P 1}$ and $R_{P 2}$ are the voltages on $C_{P 1}$ and $\mathrm{C}_{\mathrm{P} 2}$ respectively. The Kalman filter algorithm estimates the essence of the lithium-ion battery SOC by using the ampere-time integral method to calculate the SOC and uses the measured voltage value to correct the SOC value as shown in Equation (3) and (4).

$$
\begin{aligned}
& \left\{\begin{array}{l}
\operatorname{SOC}(k+1)=\operatorname{SOC}(k)-\eta \mathrm{TI}_{L}(k) / Q_{b}, \\
U_{P 1}(k+1)=U_{P 1}(k) \exp \left(-\mathrm{T} / \tau_{1}\right) \\
+R_{P 1} I_{L}(k)\left(1-\exp \left(-\mathrm{T} / \tau_{1}\right)\right. \\
U_{P 2}(k+1)=U_{P 2}(k) \exp \left(-\mathrm{T} / \tau_{2}\right) \\
+R_{P 2} I_{L}(k)\left(1-\exp \left(-\mathrm{T} / \tau_{2}\right)\right.
\end{array}\right. \\
& U_{L}(k)=U_{o c}(\operatorname{SOC}(k))-U_{P 1}(k)-U_{P 2}(k)-R_{0} I_{L}(k),
\end{aligned}
$$

Where $T$ is the sampling period; $\tau_{1}$ and $\tau_{2}$ are the time constants of the RC network, and $\tau_{1}=R_{P 1} C_{P 1}, \tau_{2}$ $=R_{P 2} C_{P 2} ; U_{P 1}$ and $U_{P 2}$ are the voltages across the RC pairs. Under equations (3) and (4), this paper selects $S O C$, $U_{P 1}$, and $U_{P 2}$ as the state variables, $U_{L}$ as the measurement variable, and $I_{L}$ as the input variable, then the state space equation is as shown in Equation (3) and (4).

\subsection{Relationship between SOC and OCV}

Open-circuit voltage (OCV) refers to the potential difference between positive and negative electrodes when the external circuit is disconnected. After a certain period of shelving, the terminal voltage of the battery is approximately equal to the open-circuit voltage. There is a relatively fixed functional relationship between the OCV and SOC, as shown in Figure 2. 


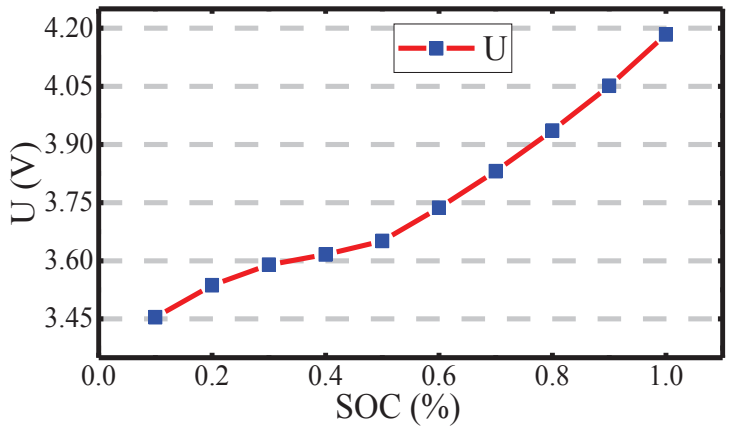

(a): OCV of the charging and the discharge process

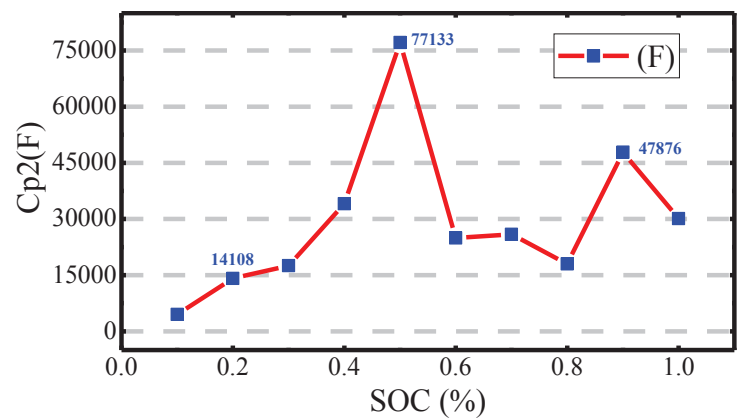

(c): Relations between SOC and $C_{P 2}$

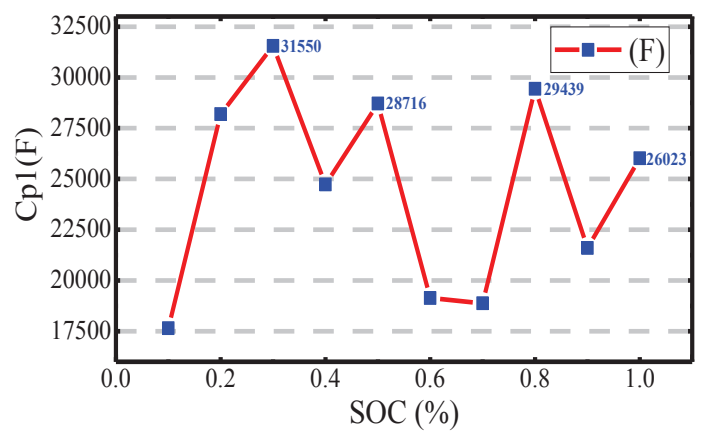

(b): Relations between $\mathrm{SOC}$ and $C_{P 1}$

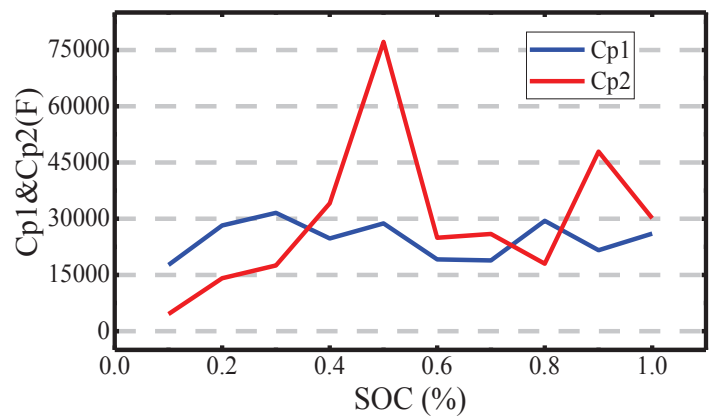

(d): Relations between SOC and $C_{P 1}-C_{P 2}$

Figure 2. Relationship between SOC and OCV

With an aging degree of temperature at a certain point, the OCV of the battery at each SOC point is measured, and then the OCV-SOC curve, which changes with SOC, is drawn. The corresponding functional relationship between $\mathrm{OCV}$ and SOC can be obtained by the curve fitting tools in MATLAB as shown in Equation (5).

$$
O C V=f(S O C)
$$

There is a functional relationship between OCV and SOC of the lithium-ion battery, so the initial SOC can be calculated by measuring the OCV. According to the principle of SOC, the initial value can be modified by the open-circuit voltage method used in Equation (5), updating and correcting the initial SOC value depends on the corresponding relationship between OCV-SOC. However, OCV-SOC relationships can be affected by external factors under actual working conditions, resulting in SOC estimation error. The OCV curve at 40, 30, 20, 10, and 0 degrees describe a more accurate OCV-SOC curve, and the results show that the improved OCV method can elevate SOC estimation effects. Also, some papers have analyzed the shelf time of the lithium-ion battery and determined that the necessary shelf time of the OCV-SOC curve is mainly influenced by internal and external factors such as battery type, SOC status, and temperature.

To avoid the serious error of SOC estimation caused by the above situation, the closed-circuit voltage method is also used to correct the initial value of SOC. The ohmic internal resistance calculation formulas are as shown in Equation (6).

$$
R_{0}=\frac{\Delta U}{I_{L}}=\frac{U_{1}-U_{2}}{I_{L}}
$$

According to the above analysis, each parameter in the high-order equivalent 2RC modeling can be identified. The corresponding weights are as shown in Equation (7).

$$
R_{P 1}=\frac{\Delta U^{\prime}}{I_{L}}=\frac{U_{4}-U_{3}}{I_{L}}
$$

The polarization capacitor $C_{P}$ in the resistance-capacitance network is charged, and the voltage decreases slowly to form a zero-input response as an expression for the RC network and this is expressed as shown in Equation (8).

$$
R_{P 2}=\frac{\Delta U^{\prime}}{I_{L}}=\frac{U_{5}-U_{4}}{I_{L}}
$$

At this time, there is a zero-input effect of the RC circuit in the circuit model. In other words, the RC circuit returns energy to the battery circuit after storing energy for a time, leading to the rise of voltage. The battery terminal voltage can be expressed as is shown in Equation (9). 


$$
U_{P}=U_{1} e^{-\frac{t}{\tau}}
$$

The circuit modeling parameters of $R_{0}, R_{P 1}, C_{P 1}, R_{P 2}$, and $C_{P 2}$ are derived from the identification results of these parameters. The values of each parameter can be obtained based on the terminal voltage that can be expressed. The open-circuit voltage calculation formula is as shown in Equation (10).

$$
U_{L}=U_{o c}-R_{0} I_{L}-R_{P 1} I_{L} e^{-t / t_{1}}-R_{P 2} I_{L} e^{-t / t_{2}}
$$

The measuring equipment used in the hybrid pulse-power characteristic test is not difficult to use, the cost of the measurement is not high, and high accuracy can be obtained. The open-circuit voltage calculation formula is as shown in Equation (11).

$$
U_{o c}=U_{P 1}-U_{P 2}-U_{P}=U_{P 1} U_{P 2}\left(1-e^{-\frac{t}{\tau}}\right)
$$

From $t 4$ to $t 2$, the ohmic internal resistance of the battery is affected, and the voltage at the battery terminal drops sharply, thereby obtaining the ohmic internal resistance of the battery. The time constant is calculated as shown in Equation (12).

$$
\tau=\frac{t_{4}-t_{2}}{\operatorname{In}\left(\frac{U_{1}-U_{4}}{U_{1}-U_{3}}\right)}
$$

In the equivalent circuit model, these equations are the values of any parameter. During the data analysis and processing, the thriving data section is derived from the initial experimental data, and then the extracted data segment is analyzed. The two-time constant $\tau_{1}$ and $\tau_{2}$ are calculated and the value of capacitor $C_{P}$ can be directly obtained as shown in Equation (13).

$$
\left\{\begin{array}{l}
\tau_{1}=R_{P 1} C_{P 1} \Rightarrow C_{P 1}=\frac{\tau}{R_{P 1}} \\
\tau_{2}=R_{P 2} C_{P 2} \Rightarrow C_{P 2}=\frac{\tau}{R_{P 2}}
\end{array}\right.
$$

As the battery voltage is its open-circuit voltage, the relationship between the OCV and the SOC can be obtained as shown in Figure(a). In this paper, the model is parameterized using a semi-automatic process that can satisfy the constraints on the optimized parameters. According to the curves in the above Figures, the value of the current and the voltage change at the time $t_{l}$ decreases sharply as it reflects the internal resistance characteristic of the high-order $2 \mathrm{RC}$ equivalent model. From time $t_{2}$ to $t_{3}$ and also $t_{4}$ to $t_{5}$ the process from Figure $2(\mathrm{~b})$ shows that the value of the terminal voltage $U_{L}$ gradually decreases. According to the KVL of the high-order $2 \mathrm{RC}$ equivalent model, the polarization of the $C_{P 1}$ and $C_{P 2}$ gradually reduces the terminal voltage, and the reverse polarization known as the polarization voltage $U_{L}$ gradually increases and finally equals the open-circuit voltage from $0 \mathrm{~V}$.

Finally, the voltage and current curves of the whole HPPC experimental pulse charge and discharge process correspond to the High-order 2RC equivalent model showing the polarization effect of the battery. There are many commonly used SOC estimation methods, among which the error of the ampere-time integral method is large. The open-circuit voltage method is mainly used for offline estimation, as well as the high-precision SOC estimation methods such as the neural network method, fuzzy reasoning method, and the Kalman filtering method.

\subsection{Reduced-order EKF algorithms}

\subsubsection{Kalman filter algorithm}

The principles of the Kalman Filter (KF) algorithm are one of the most widely used intelligent algorithms and are often used in practical scenarios such as path planning, target tracking, and SOC estimation of lithium batteries [49]. The measurement update process also called the calibration process is a method of feeding back the observations and correcting the deviations. The Kalman filter algorithm is an optimized autoregressive data processing algorithm that is not only suitable for stationary processes but also non-stationary processes with good real-time performance and easy implementation. Its state equations and observation equations are as shown in Equations 14

$$
\left\{\begin{array}{l}
X_{k}=N_{k-1} x_{k-1}+M_{k-1} u_{k-1}+V_{k-1} \\
s_{k}-1=G_{k-1} x_{k}+H_{k} u_{k}+\omega_{k}
\end{array}\right.
$$

Where $X_{k}$ represents the system state variable at a time $k, s_{k}$ is the system observed variable at a time $k, U_{k}$ is 
the system input which is used as the control variable; $N_{k}$ is the transfer matrix of state $\mathrm{x}$ from ${ }^{k-1}$ to $k$, and $M_{k}$ is the input matrix. $G_{k}$ is the measurement matrix, $H_{k}$ is the feedforward matrix; $\omega k$ is the noise of the system state equation i.e., the process noise, whose variance is $Q_{k}, v_{k}$ is the noise of the measurement equation i.e., the observed noise, whose variance is $R_{k}$.

\subsubsection{Reduced-order EKF algorithm}

The lithium-ion battery is a dynamic nonlinear system and a reduced-order extended Kalman filter has been considered a standard selection in the nonlinear state estimation. Reduced-order EKF is the nonlinear version of the Kalman filter which linearizes an estimate of the current mean and covariance [50]. When the precise statespace model for batteries is established, the EKF method is capable of keeping track of the estimated state of the system and the variance or uncertainty of the estimate in case the accurate initial state is given. Otherwise, the convergence will cost more time and even be lost sometimes. If given an effective search mechanism to access the original states, this unfulfilled situation can be improved. After obtaining the linearization model, the SOC of the lithium-ion battery is further estimated by the Kalman filter, and the algorithm flow of the extended Kalman is shown in Figure 3.

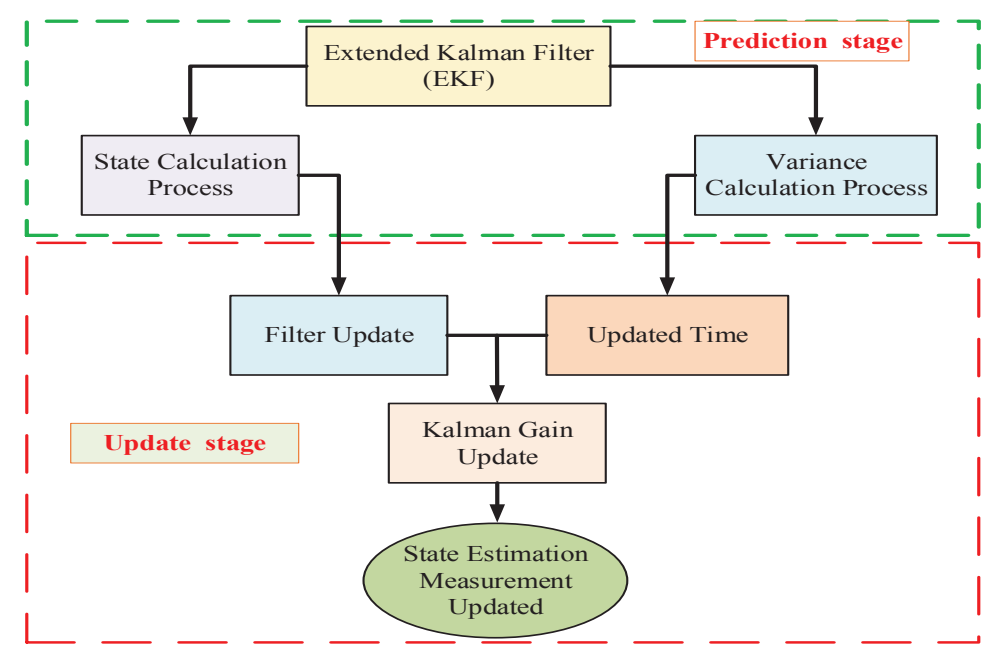

Figure 3. Reduced-order EKF-based implementation process

In the case of linearization error, the value calculated by the mathematical model and the physical value directly measured by the physical method and the measured true value is equal because of the linearization error of the mathematical model plus the prior estimate. There is a certain error between the value and the estimated value. There is an error between all the measured values obtained by the mathematical model and the actual measured true values. The extended Kalman filter algorithm is based on the ordinary Kalman filter algorithm.

Extended Kalman filtering is a technique for online linearization [51]: This means linearizing the estimated parameters and then performing linear Kalman filtering thereby achieving a SOC estimation of the lithium-ion battery. In this case, system noise and process noise are generally approximated as white noise under Gaussian distribution. The expression equations and observation equations of the discrete nonlinear system space are as shown in Equation (15)..

$$
\left\{\begin{array}{l}
X_{k+1}=f\left(X_{k}, k\right)+\omega k \\
Z_{k}=h\left(X_{k}, k\right)+v_{k}
\end{array}\right.
$$

Where $f(*)$ is the system function, $\mathrm{h}(*)$ is the output function, both of them are nonlinear functions that could be linearized by the first-order Taylor series. Here $X_{K+1}$ represents the state vector, $X_{k}$ is the n-dimensional system state, $K$ stands for the discrete vector, $\omega_{k}$ is the process noise vector, and modeling errors $Z_{K}$ in the $\mathrm{m}$ dimensional observation vector and $v_{k}$ is the measurement noise. $\omega_{k}$ and $v_{k}$ are usually considered as the zero-mean Gaussian white noises. Further computations on the formulas can be seen as shown in Equation (16). 


$$
\left\{\begin{array}{l}
\left.f\left(X_{k}, k\right) \approx f\left(X_{k}, k\right) f \frac{\partial f\left(X_{k}, k\right)}{\partial X_{k}}\right|_{X_{k} \approx X_{k}}\left(X_{k} \approx X_{k}\right) \\
\left.h\left(X_{k}, k\right) \approx h\left(X_{k}, k\right) f \frac{\partial h\left(X_{k}, k\right)}{\partial X_{k}}\right|_{X_{k} \approx X_{k}}\left(X_{k} \approx X_{k}\right)
\end{array}\right.
$$

To avoid the high-order term for updating the linear space equation, the current state transformation matrix and observation driving matrix can be achieved. Centered on the linear system's spatial equation, the extended Kalman filtering principle is simply applied to equation (16). and the values of $E_{k}, F_{k}, G_{k}$, and $H_{k}$ are expressed as shown in Equation (17).

$$
\left\{\begin{array}{l}
E_{k}=\left.\partial f \frac{\left(X_{k}, k\right)}{\partial X_{k}}\right|_{X_{k} \approx X_{k}} \\
F_{k}=f\left(X_{k}, k\right)-E_{k}=X_{k} \\
G_{k}=\left.\frac{\partial h\left(X_{k}, k\right)}{\partial X_{k}}\right|_{X_{k} \approx X_{k}} \\
H_{k}=h\left(X_{k}, k\right)-G_{k}=X_{k}
\end{array}\right.
$$

Further mathematical computations can be applied to linearize the formula as shown in Equation (18).

$$
\left\{\begin{array}{l}
X_{k+1}=E_{k} X_{k}+F_{k}+w_{k} \\
Z_{k}=G_{k} X_{k}+H_{k}+v_{k}
\end{array}\right.
$$

The recursive process of the extended Kalman filter algorithm formula is obtained by applying the Kalman filter basic equation as shown in Equation(19).

$$
\left\{\begin{array}{l}
X_{k}^{-}=E X_{k-1}+F I_{I ., k-1} \\
P_{k \mid k-1}=E P_{k-1} E^{T}+Q_{k} \\
K_{k}=P_{k \mid k-1} G^{T}\left(G P_{k \mid k-1} G^{T}+R_{k}\right) \\
X_{k}=X_{k}^{-}+K_{k}\left(U_{I ., k}-G X_{k-1}-H I_{I ., k-1}\right) \\
P_{k}=P_{k \mid k-1}-K_{k} P_{k \mid k-1}
\end{array}\right.
$$

Where ${ }^{X_{k}^{-}}$is the direct estimate time $k,{ }^{X_{k-1}}$ is the optimal estimate state value at the last moment. ${ }_{k \mid k-1}$ is the covariance of $X_{k}^{-}, Q_{k}$ is the covariance of $w_{k}$. $K_{k}$ is the Kalman gain. The SOC estimation of the battery is an obvious hidden Markov model.

\section{Experimental verification analysis}

\subsection{Implementation of the test platform}

All the data in this paper was derived from the comparison and verification of the HPPC test on the ternary $3.7 \mathrm{~V}$ 70Ah NCM Prismatic lithium-ion battery for parameter identification at $25^{\circ} \mathrm{C}$, capacity calibration for constant current charge and discharge, and algorithm complexity for BBDST conditions. The battery test equipment used was the BTS750-200-100-4 and the incubator used for the temperature was the BTT-331C. The experimental platform used for the experiment is shown in Figure 4 


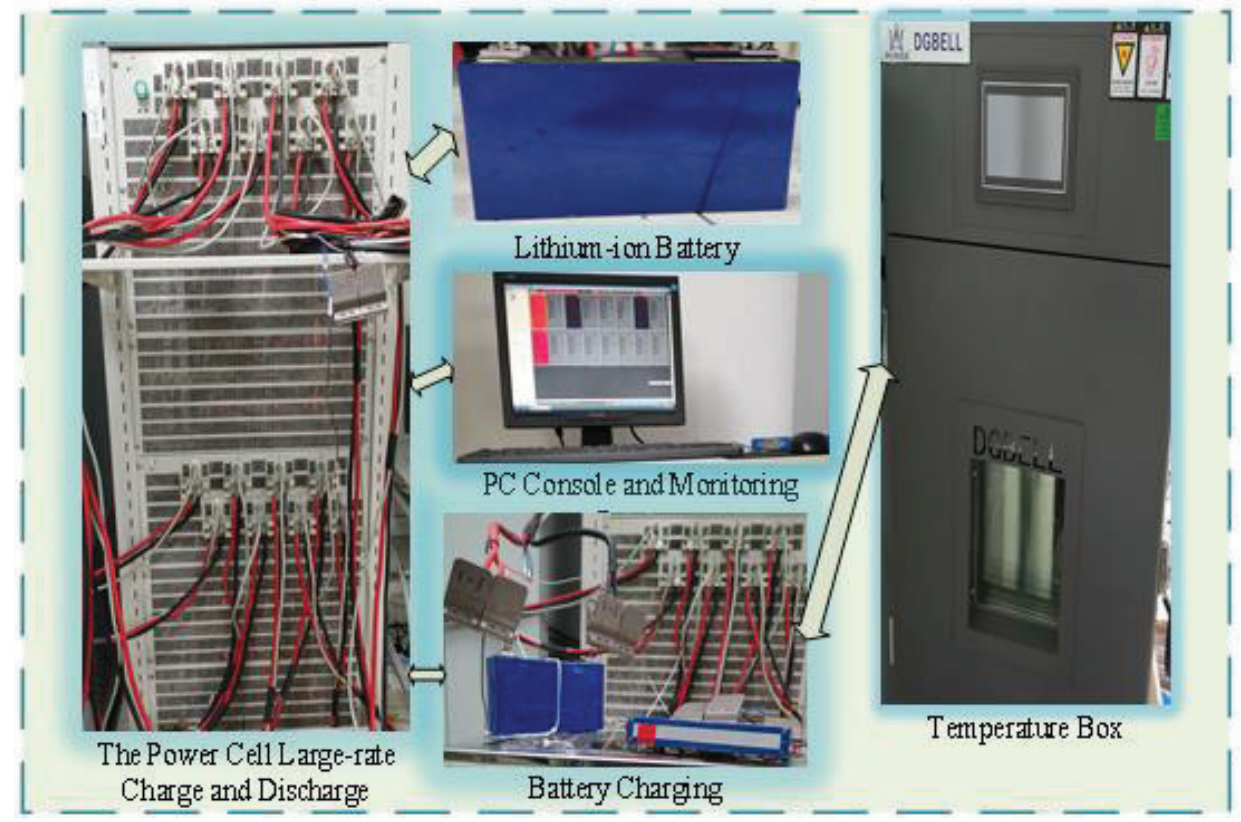

Figure 4. The experimental platform

The battery will age due to recycling and other reasons, and the actual capacity of the battery will have greatly deviated from the calibration capacity of 70Ah. The true discharge capacity of the battery is important for the estimation of the SOC of the lithium-ion battery. Therefore, the capacity calibration of the battery must first be performed. In this study, the parameter identification will increase the complexity of the algorithm but the accuracy is not improved. The battery is subjected to a Pulse discharge experiment, and the battery model parameters are obtained by analyzing the operating characteristics of the battery during operation. The specifications of the ternary 3.7V 70Ah NCM Prismatic lithium-ion battery are shown in Table 2.

Table 2. Basic technical parameters of the lithium-ion battery

\begin{tabular}{cc}
\hline Criteria/ Factors & Parameters \\
\hline Cell nominal capacity/Ah & 70 \\
Rated voltage/V & 3.7 \\
Charge cut-off voltage/V & $4.2 \pm 0.05$ \\
Discharge cut-off voltage/ $\mathbf{~}$ & $2.75 \pm 0.05$ \\
Size: length * width * height/mm & $14 \times 38 \times 91$ \\
Standard charge current & $1 \mathrm{C}$ \\
Standard discharge current & $2 \mathrm{C}$ \\
Maximum load current & $3 \mathrm{C}$ \\
Internal resistance/m $\Omega$ & $0.5-1.0$ \\
Working temperature/ $\square$ & $-10-+35$ \\
\hline
\end{tabular}

\subsection{Experimental process and parameters identification}

The essential part of the parameter measurement process of the battery model is model-based SOC estimation. After determining the structure of the battery model, the model parameters need to be identified. As shown in Figure 1, the parameters, including $U_{O C}(S O C)$, ohmic resistance $R_{0}$, polarization capacitance $C_{P 1}$, and $C_{P 2}$, and polarization resistance $R_{P 1}$ and $R_{P 2}$, should be determined. Therefore, the following experimental processes are conducted on the lithium-ion battery with a nominal capacity of 70Ah and the identification steps are as follows:

1) Set the ambient temperature for the battery to $25^{\circ} \mathrm{C}$.

2) Fully charge the battery at constant current and constant voltage (CC-CV) until the charging current drops to $0.05 \mathrm{C}$.

3) The lithium-ion battery with $\mathrm{SOC}=1$ was discharged at a rate of $1 \mathrm{C}$ for 6 minutes $(\mathrm{SOC}=0.9)$ and left for 40 minutes, and then the HPPC experiment was performed, and the open-circuit voltage, the discharge current, and the discharge times were recorded.

4) Discharge by $2 \%$ SOC with $1 / 3$ C-rate to adjust the SOC 
5) Rest the battery for $2 \mathrm{~h}$ and record the voltage characteristic.

6) Repeat steps (4) and (5) until SOC $=1 \%$ or discharge cut-off voltage is reached.

According to the above experimental steps, the lithium-ion battery capacity is first calibrated as $70 \mathrm{Ah}$ at $25^{\circ} \mathrm{C}$ and then the pulse discharge test is conducted on the battery. With the HPPC experimental data, the corresponding relationship between different SOC points and various parameters can be achieved for the high-order $2 \mathrm{RC}$ equivalent circuit model at $25^{\circ} \mathrm{C}$ as shown in

\begin{tabular}{|c|c|c|c|c|c|c|c|}
\hline$S O C$ & $\mathrm{UOC} / \mathrm{OCV}$ & $R 0 / \Omega$ & $R p 1 / \Omega$ & $R p 2 / \Omega$ & $C p 1 / F$ & $C p 2 / F$ & $I$ \\
\hline 1 & 4.184 & 0.001311429 & $3.33857 \mathrm{E}-05$ & 0.000468286 & 26023.10655 & 30131.17755 & 70 \\
\hline 0.9 & 4.0513 & 0.001302857 & $9.28571 \mathrm{E}-05$ & 0.051257143 & 21603.07692 & 47876.25418 & 70 \\
\hline 0.8 & 3.936 & 0.001302857 & 0.000716714 & 5.37714E-05 & 29439.90433 & 17998.40595 & 70 \\
\hline 0.7 & 3.8309 & 0.001302857 & 4.29143E-05 & 0.000639 & 18877.16378 & 25931.14241 & 70 \\
\hline 0.6 & 3.7366 & 0.001305714 & 4.33429E-05 & 0.000623429 & 19138.10152 & 24926.67278 & 70 \\
\hline 0.5 & 3.6511 & 0.001311429 & 0.000301571 & 1.37943E-05 & 28716.24822 & 77133.38857 & 70 \\
\hline 0.4 & 3.6163 & 0.001324286 & $2.46571 \mathrm{E}-05$ & 0.000365714 & 24727.11472 & 34070.3125 & 70 \\
\hline 0.3 & 3.59 & 0.001351429 & 0.000376857 & 2.88857E-05 & 31550.41698 & 17531.15727 & 70 \\
\hline 0.2 & 3.5369 & 0.001398571 & 0.000468857 & 3.77143E-05 & 28196.22182 & 14108.71212 & 70 \\
\hline 0.1 & 3.4545 & 0.001478571 & 0.000696714 & 0.000101271 & 17654.29567 & 4532.374101 & 70 \\
\hline
\end{tabular}

Table 3. Variation of parameters at different SOC points

\begin{tabular}{|c|c|c|c|c|c|c|c|}
\hline SOC & $\mathrm{UOC} / \mathrm{OCV}$ & $R 0 / \Omega$ & $R p 1 / \Omega$ & $R p 2 / \Omega$ & $C p 1 / F$ & $C p 2 / F$ & $I$ \\
\hline 1 & 4.184 & 0.001311429 & 3.33857E-05 & 0.000468286 & 26023.10655 & 30131.17755 & 70 \\
\hline 0.9 & 4.0513 & 0.001302857 & $9.28571 \mathrm{E}-05$ & 0.051257143 & 21603.07692 & 47876.25418 & 70 \\
\hline 0.8 & 3.936 & 0.001302857 & 0.000716714 & 5.37714E-05 & 29439.90433 & 17998.40595 & 70 \\
\hline 0.7 & 3.8309 & 0.001302857 & 4.29143E-05 & 0.000639 & 18877.16378 & 25931.14241 & 70 \\
\hline 0.6 & 3.7366 & 0.001305714 & 4.33429E-05 & 0.000623429 & 19138.10152 & 24926.67278 & 70 \\
\hline 0.5 & 3.6511 & 0.001311429 & 0.000301571 & 1.37943E-05 & 28716.24822 & 77133.38857 & 70 \\
\hline 0.4 & 3.6163 & 0.001324286 & $2.46571 \mathrm{E}-05$ & 0.000365714 & 24727.11472 & 34070.3125 & 70 \\
\hline 0.3 & 3.59 & 0.001351429 & 0.000376857 & $2.88857 \mathrm{E}-05$ & 31550.41698 & 17531.15727 & 70 \\
\hline 0.2 & 3.5369 & 0.001398571 & 0.000468857 & $3.77143 \mathrm{E}-05$ & 28196.22182 & 14108.71212 & 70 \\
\hline 0.1 & 3.4545 & 0.001478571 & 0.000696714 & 0.000101271 & 17654.29567 & 4532.374101 & 70 \\
\hline
\end{tabular}

The voltage source $U_{O C}$ is utilized to describe the nonlinear monotonous relationship between OCV and SOC, which can be denoted as $U_{O C}$ (SOC); the $R_{0}$ is the ohmic resistance of the lithium-ion battery, which represents the ohmic polarization; in this case, $R_{P 1}$ and $C_{P 1}$ are concentration resistance and concentration polarization resistance respectively, which describe the long-term polarization response; $R_{P 2}$ and $C_{P 2}$ are electrochemical polarization resistance and electrochemical polarization capacitance respectively, which describe the short-time polarization response; ${ }^{I}$ and $U_{L}$ are the load current and terminal voltage, respectively. 


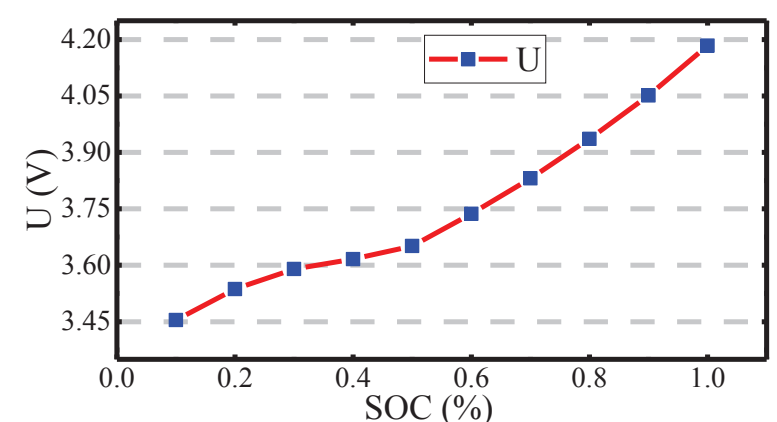

(a): OCV-SOC variation curve of the charging and the discharge process

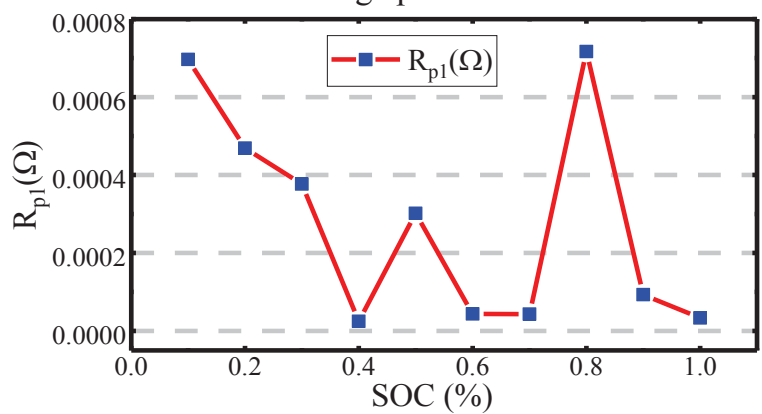

(c): The Relation between $\mathrm{SOC}$ and $R_{P 1}$

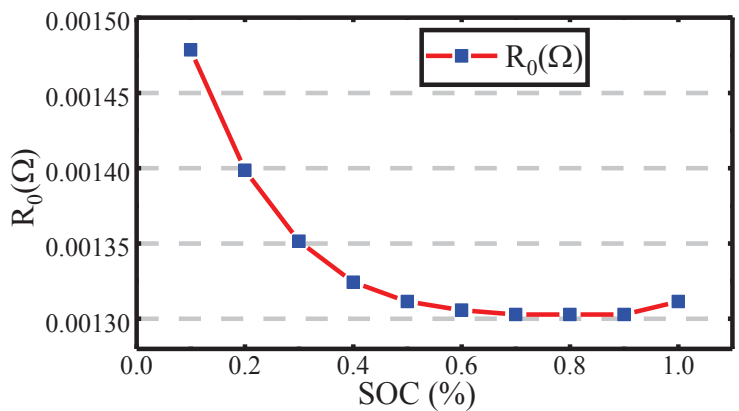

(b): The Relation between SOC and $R_{0}$

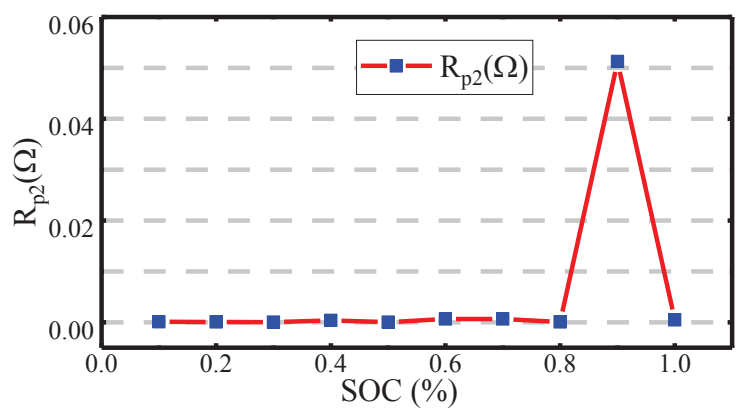

(d): The Relation between SOC and $R_{P 2}$

Figure 5. Identification results of RC network parameters

The fitted parameter is then shown with a polynomial of the 6th order, and the following results are obtained. The fitting results of the curve are $R_{0}, R_{P 1}, R_{P 2}, C_{P 1}$, and $C_{P 2}$ respectively, reflecting the parameters for the equivalent circuit model. The fitted results are shown in the following Equation (20).

$$
\begin{aligned}
& \left\{\begin{array}{l}
\left\{\begin{array}{l}
f\left(U_{o c}\right)=19.92 * u^{6}-60.68 * u^{5}+67.37 * u^{4} \\
-31.55 * u^{3}+5.05 * u^{2}+0.7097 * u+3.358
\end{array}\right. \\
\left\{\begin{array}{l}
f\left(R_{0}\right)=0.001588 * u^{6}-0.005002 * u^{5}+0.007021 * u^{4}- \\
0.00629 * u^{3}+0.00405 * u^{2}-0.001665 * u+0.00161
\end{array}\right. \\
\left\{\begin{array}{l}
f\left(R_{p 1}\right)=-4.715 * 10^{7} * u^{6}+1.464 * 10^{8} * u^{5}-1.731 * 10^{7} * u^{4}+ \\
9.773 * 10^{7} * u^{3}-2.717 * 10^{7} * u^{2}+3.555 * 10^{6} * u-1.607 * 10^{5}
\end{array}\right. \\
\left\{\begin{array}{l}
f\left(R_{p 2}\right)=-38.94 * u^{6}+119.4 * u^{5}-141.4 * u^{4}+81.68 * u^{3}-23.81 * u^{2} \\
+3.237 * u-0.1548
\end{array}\right. \\
\left\{\begin{array}{l}
f\left(C_{p 1}\right)=4.995 * 10^{6} * u^{6}-1.625 * 10^{7} * u^{5}+1.997 * 10^{7} * u^{4} \\
1.131 * 10^{7} * u^{3}+2.802 * 10^{6} * u^{2}-2.031 * 10^{5} * u+1.958 * 10^{4}
\end{array}\right. \\
\left\{\begin{array}{l}
f\left(C_{p 2}\right)=-4.715 * 10^{7} * u^{6}+1.464 * 10^{8} * u^{5}-1.731 * 10^{7} * u^{4}+ \\
9.773 * 10^{7} * u^{3}-2.717 * 10^{7} * u^{2}+3.555 * 10^{6} * u-1.607 * 10^{5}
\end{array}\right.
\end{array}\right.
\end{aligned}
$$

\subsection{HPPC test results and parameter Identification}

The variation of the model parameters can be attained and embedded into the state-space equation for the subsequent SOC estimation by the curve fitting for the ECM model and the circuit voltage relationship expression between $U_{O C}$ and SOC. The cure variations of current and voltage are presented in Figure 6 


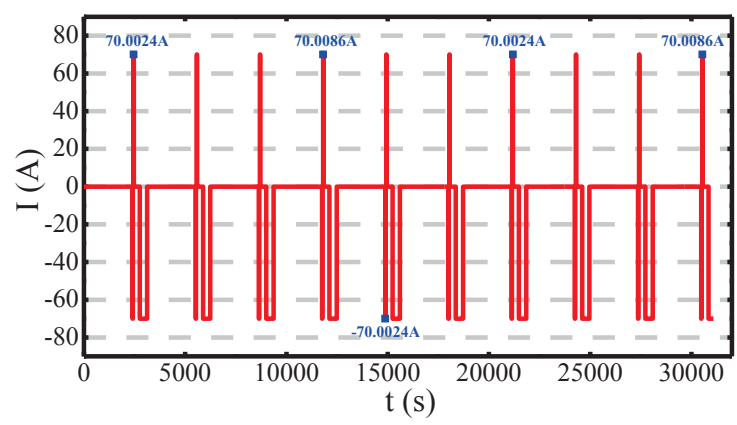

(a): Complete HPPC current variation

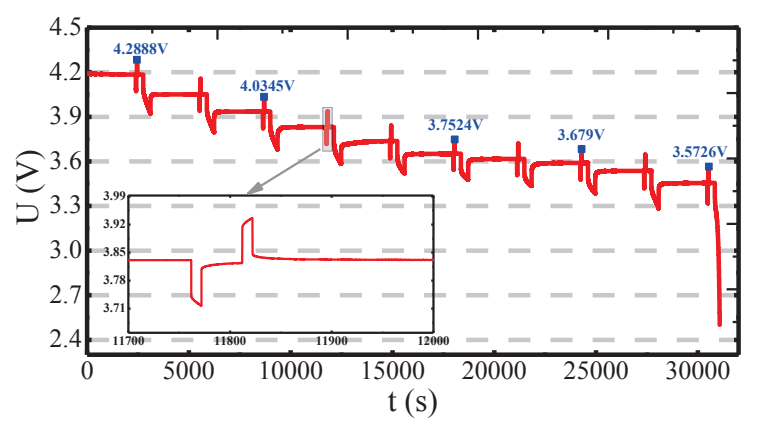

(c): Complete HPPC voltage response variation

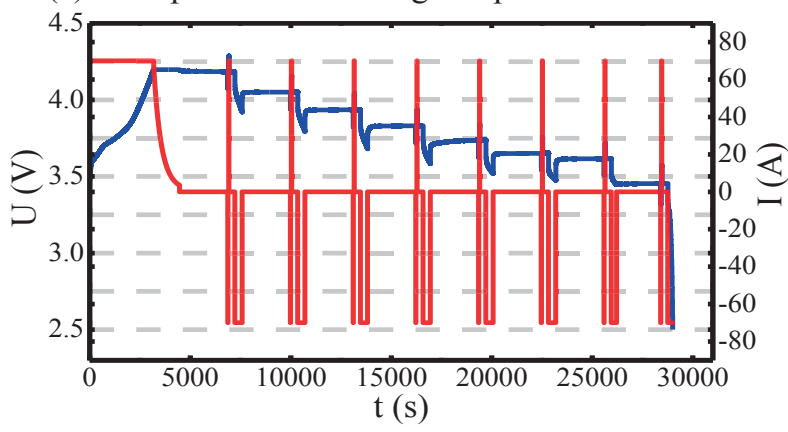

(e): Complete voltage and current cycle variation

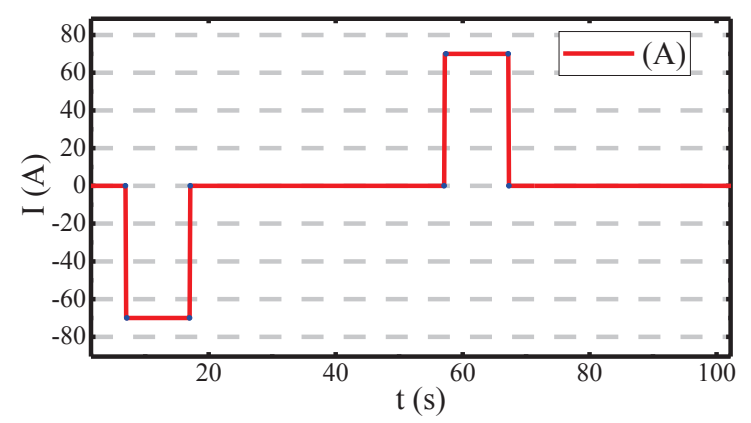

(b): Partial HPPC current variation

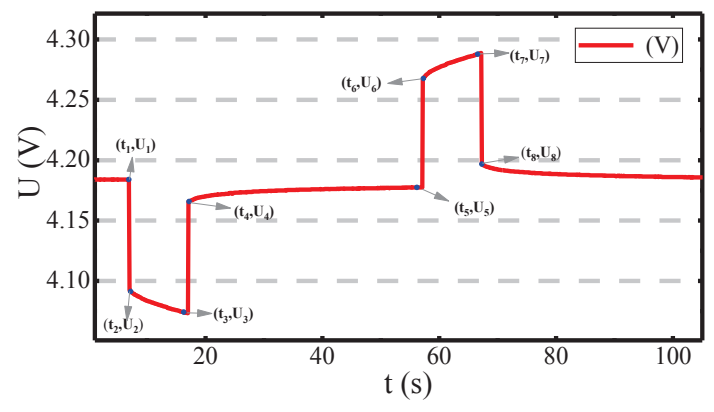

(d): Partial HPPC voltage response variation

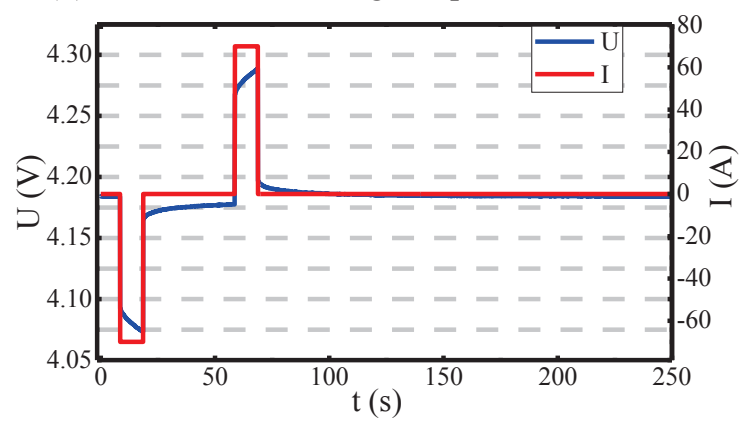

(f): Partial-cycle voltage and current variation

Figure 6. HPPC Pulse discharge test index curves

The complete curves of current and terminal voltage are shown in Figure 6 (a), and Figure 6 (c) respectively. The current and voltage curve of one of the pulses in the test is shown in Figure 6 (b), and Figure 6 (d), respectively. The battery voltage will gradually stabilize after a long period at the end of discharge, which means that the internal chemical reaction and thermal effects have reached equilibrium. The model is very related to the actual battery characteristics, so making it simple and easy to use. Parameter identification can be accomplished by establishing an equation for the zero-state and zero-input states of the High-order 2RC equivalent model. From the voltage response curve of the HPPC test experiment battery, the characteristics of each SOC point can be obtained. By analyzing Figure 6(d), the following conclusions can follow:

1) U1 U 2 : The voltage transient is caused by the ohmic internal resistance $R_{0}$, and the voltage drops from $\mathrm{U} 1$ to $\mathrm{U} 2$.

2) U2 U3: The polarization capacitor $C_{P 1}$ in the RC network is charged, and the voltage is slowly decreased to form a zero-input response;

3) U3 U4: At this time point, the current is abrupt, the current suddenly changes to 0 , and the voltage drop on the ohm internal resistance disappears, causing the voltage transient to rise;

4) U4 U5: At this time, the polarization capacitor $C_{P 2}$ is discharged through the polarization of internal resistance $R_{P 2}$, and the voltage slowly rises.

The voltage of the battery terminal at $t_{1}-t_{2}$ drops instantaneously because the battery's discharge current increases abruptly from 0 to $1 \mathrm{C}$ resulting in a sudden change in voltage on the ohmic internal resistance. The terminal voltage of the $t_{2}-t_{3}$ battery decreases slowly during the process of discharging and the current charging polarization capacitor is caused by the zero-state response of the $2 \mathrm{RC}$ loop. The terminal voltage of $t_{3}-t_{4}$ the battery 
rises instantaneously, which is caused by the sudden change in the discharge current to 0 and the disappearance of voltage on the ohmic internal resistance. In the period $t_{4}-t_{5}$, the slow rise in the battery terminal voltage is the process of polarization resistance discharge by the polarization capacitor which is caused by the zero-input response of the $2 \mathrm{RC}$ circuit. $t_{5}$ time is the charging phase of the battery. There is a phase of sudden voltage increase and a phase of slow voltage increase during the charging. In the study of this experiment, the values $R_{0}, R_{P 1}, R_{P 2}$, and $C_{P 1}, C_{P 2}$ were all substituted into the algorithm in a mean way. The EKF estimation of the lithium-ion battery $\mathrm{SOC}$ algorithm was verified by $0.5 \mathrm{C}$ constant current discharge and combined equations.

\subsection{Simulation verification}

After identifying the parameters of the model, the dynamic simulation model of the equivalent circuit was constructed in Simulink/Matlab. The identified parameters were put into the simulation model and in addition to different working currents. The output voltage response of the model was compared with the actual voltage data, and the model was verified. To evaluate the accuracy of the model parameters, the variable operating current is added to the model and the difference between the model output voltage and the time output voltage is compared. The model established in Simulink/Matlab is as shown in Figure 7.

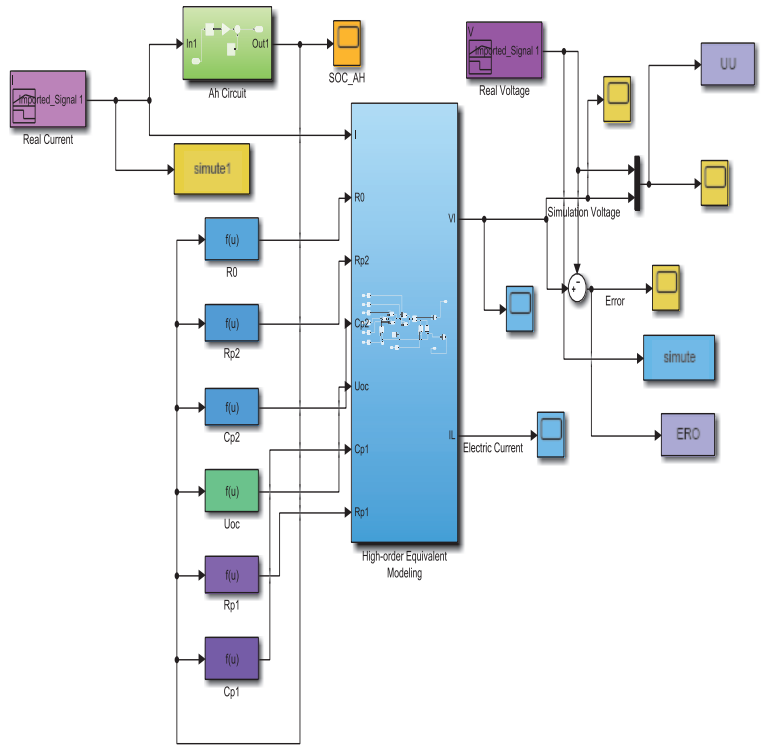

(a): The Simulation Model of the $2 \mathrm{RC}$ equivalent model

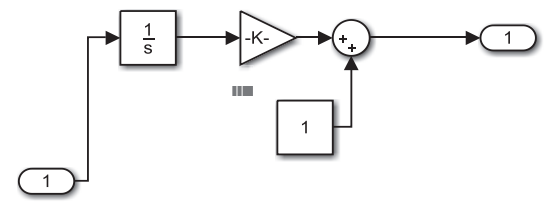

(b): Inner structure of ampere-hour (Ah) model

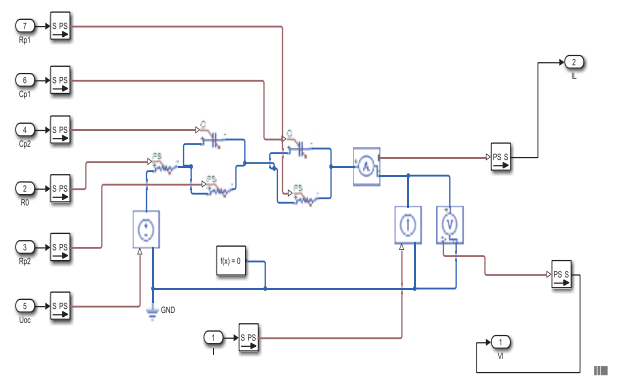

(c): The internal circuitry of the $2 \mathrm{RC}$ equivalent model

Figure 7. Structural diagram of the Simulink verification model

The Simulink simulation structure established is to calculate SOC with discharge current, and the amperehour $(\mathrm{Ah})$ integral method is applied to its internal structure. In the internal structure of the high-order 2RC equivalent model, each circuit component is a controllable parameter that changes over time. The model has five inputs and one output. The inputs are $I, R_{0}, R_{P 1}, C_{P 1}, R_{P 2}, C_{P 2}$, and $U_{O C}$ and the output is the terminal voltage U. This model can simulate the working condition of the lithium-ion battery.

Considering that the battery is often in an intermittent discharge state in actual use, the model is further simulated and analyzed under the conditions of cyclic discharge shelving. The experimental results are shown in Figure(a), and the estimated deviation is shown in Figure 8 (b).

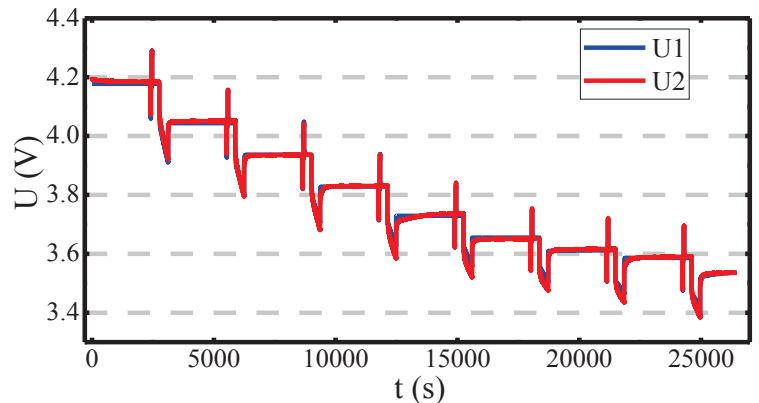

(a): Comparison of experimental and simulated voltage

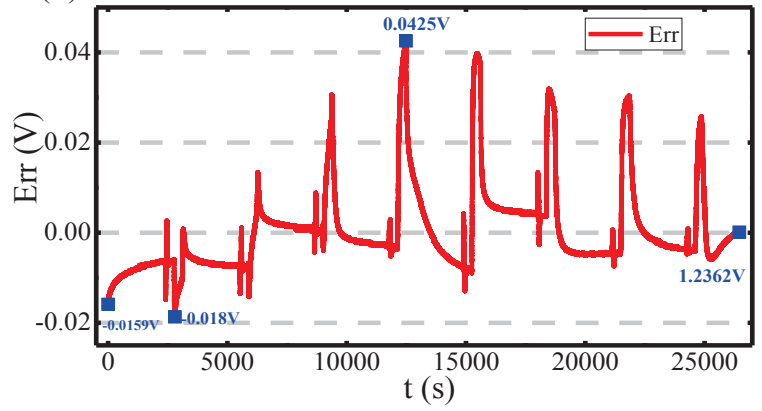

(b): Voltage error curve

Figure 8. Experimental and simulated voltage variations 
The difference between the model output voltage and the original battery output voltage is described in Figure(b), as the voltage error. The Figure shows a powerful tracking effect on the original value of the expected value. The calculated average deviation is approximately $0.0425 \mathrm{~V}$, which can explain the voltage value of the battery on the operating end. And the voltage measurement deviation increases at the end of the battery discharge by studying the voltage comparison error between the two. The battery voltages vary greatly before the final discharge in this regard. The simulation outcome is followed by a measurement error. The precision of the model is $99.14 \%$, while $4.4 \mathrm{~V}$ is the maximum lithium battery voltage.

\subsection{Dynamic test condition analysis}

The Beijing Bus Dynamic Stress Test (BBDST) setting working condition test is used to test the lithium-ion battery. The BTS770-200-100-4 battery testing equipment provided by Shenzhen Yakeyuan Technology Co., Ltd. is used for battery charging and discharging.

The BBDST working condition is the actual data acquisition of the Beijing bus. In Table $1, \mathrm{Ph}(\mathrm{kW})$ is the actual battery output power under the conditions of the bus start acceleration and taxiing. The data in $\operatorname{Pc}(\mathrm{W})$ was obtained by reducing $\mathrm{Ph}(\mathrm{kW})$, and $\mathrm{Pc}(\mathrm{W})$ was used for the experiment of the ternary 3.7V 70Ah NCM Prismatic lithium-ion battery of China National Aviation Corporation. It can be seen from Table 1 that the time of a complete BBDST is 300s, and the BBDST condition test is performed 20 times on the battery, and the BBDST condition data obtained is as shown in Figure 9

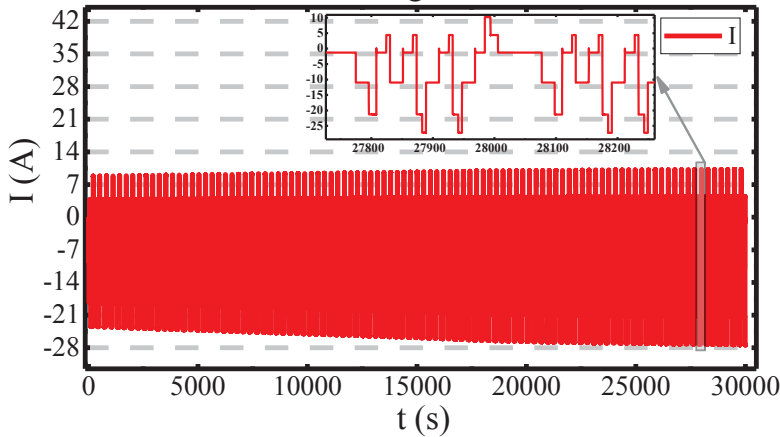

(a): BBDST working condition current

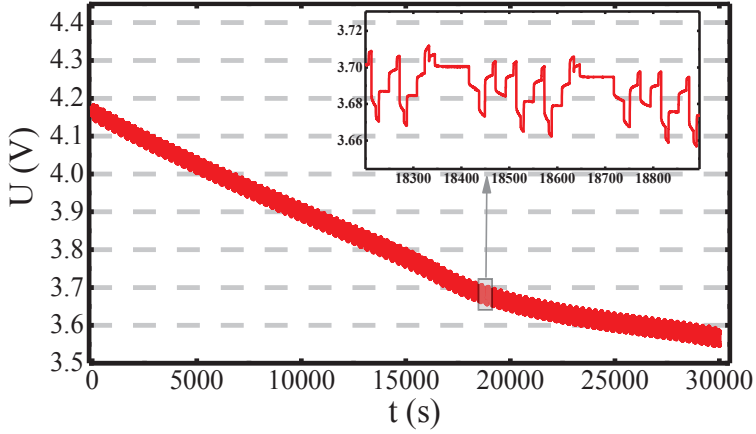

(b): BBDST working condition voltage

Figure 9. BBDST working condition experimental data

Figure 9 (a) and (b), are the experimental current and voltage data of BBDST conditions respectively. Since the BBDST operating condition is power discharged, it can be seen from Figure(a), that as the number of cycles increases, the discharge current increases, and according to Figure 9(b), the battery terminal voltage as a whole shows a downward trend.

The High-order 2RC equivalent modeling was established by parameter identification of HPPC experimental data. To verify the validity of the model, the model data and actual data were compared and analyzed with additional battery operating conditions data. The model was verified by the BBDST condition, and the various working conditions of the battery were simulated by constant power discharge for a certain period. The test device obtained the current value in the experimental data as the input condition, and the simulated terminal voltage is compared with the experimental acquisition terminal voltage by the simulation model, and the result is as shown in Figure.

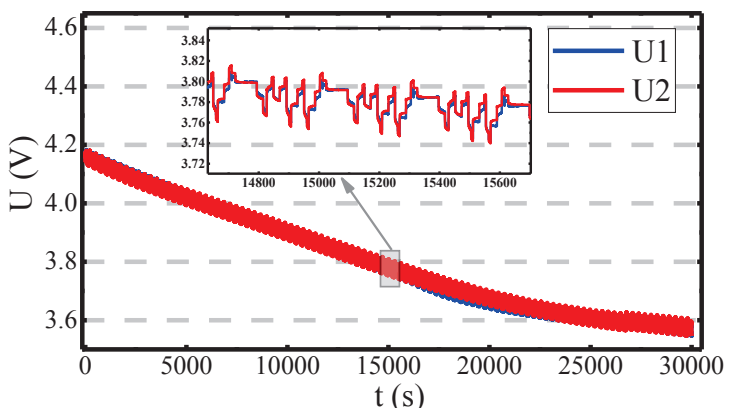

(a): Simulation terminal voltages

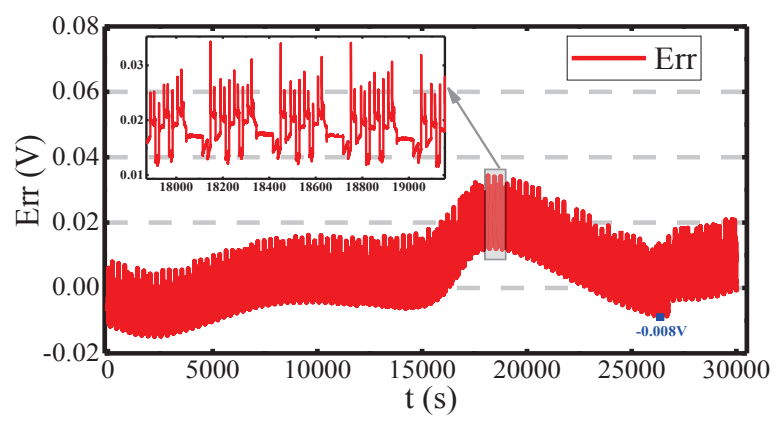

(b): Simulation terminal voltage Error

Figure 10. Simulation terminal voltage and experimental terminal voltage comparison chart

In Figure, U1 is the real terminal voltage data curve obtained by the test equipment, and U2 is the output terminal voltage curve obtained by the simulation model under the input current condition. It can be seen from Figure 10 that the simulation curve and the actual test curve have similar trends, which can better simulate the battery discharge. According to the established high-order 2RC equivalent mode and the reduced-order extended 
Kalman filter algorithm, the SOC estimation of the BBDST condition is carried out. The error between the initial value and the true value is given at least $20 \%$ in advance so that the SOC estimation is performed, and the obtained result is shown in Figure.

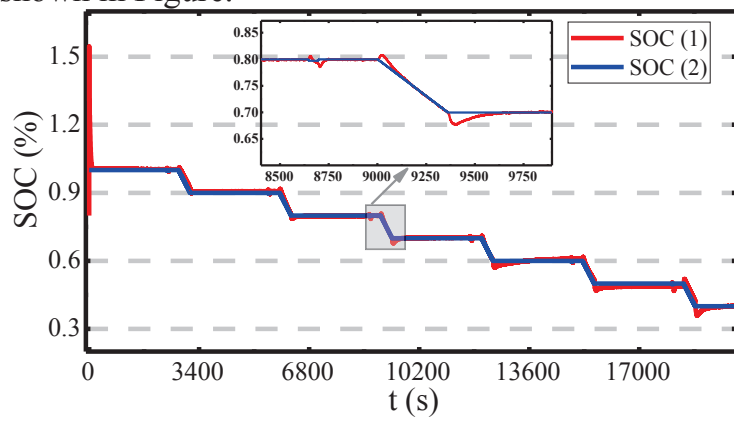

(a): Comparison of SOC estimation result

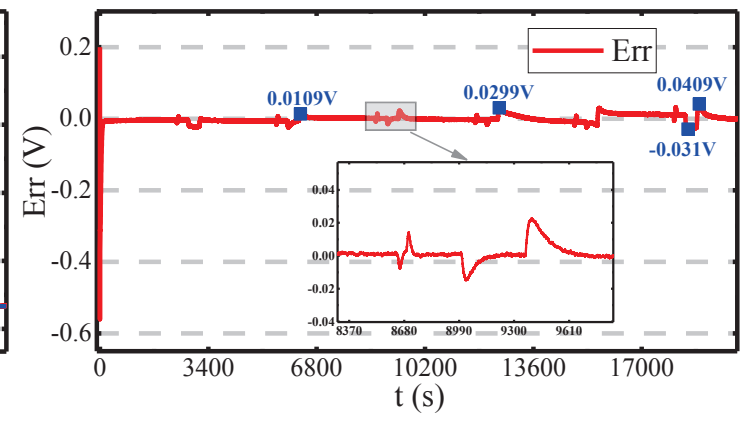

(b): SOC estimation Error

Figure 11. Comparison of SOC variation curves

In Figure(a), SOC1 is the true SOC value, and SOC2 is the SOC estimation value using a REKF algorithm. Figure(b) is the REKF error curve obtained the difference in the two SOC value curves. Figure(b), shows the error of SOC estimation based on the established high-order 2RC equivalent modeling using the reduced-order extended Kalman filter. The Figure shows the theoretical calculation of the battery SOC and the estimated value of the reduced-order EKF algorithm. It can be seen from the Figure that the reduced-order EKF algorithm converges quickly and the tracking effect is good enabling the initial algorithm to converge within the required time of about 100 s to track the theoretical value. It can also be observed that the estimated deviation is stable within $-0.031 \mathrm{~V}$ and $0.0409 \mathrm{~V}$, and the overall performance is excellent. It is proven that the reduced-order EKF has good convergence and has optimum tracking performance for SOC estimation. The algorithm can correct the error of the initial value very well, does not depend on the accuracy of the initial value, and has a strong correction function. However, the maximum error of the SOC estimation at the end of the discharge of the method described herein is $1.85 \%$

\section{Conclusions}

In this study, the high-order $2 \mathrm{RC}$ equivalent model is selected to identify the parameters. Through detailed circuit analysis, the accurate expressions of the $2 \mathrm{RC}$ time constant and terminal voltage of lithium batteries are derived. After HPPC-Reduced-order EKF experiments, the parameters are accurately identified and a Simulink model is established in MATLAB to verified by simulation with the HPPC experimental data. The parameter identification method adopted in this paper can make the accuracy of the equivalent circuit model reach more than $98.15 \%$ accuracy. The research content of this paper can provide an accurate parameter identification method for the lithium-ion battery mathematical model, and serve as an important theoretical basis for the accurate estimation of SOC in a battery management system. Moreover, based on the linearization method adopted, the high-order 2RC equivalent model and the reduced-order extended Kalman filter is constructed for the SOC estimation algorithm of LIB. Then the effectiveness of this algorithm is verified experimentally through fully and partially charged battery discharge experiments.

\section{References}

1. Chen, Z., et al., Particle filter-based state-of-charge estimation and remaining-dischargeable-time prediction method for lithium-ion batteries. Journal of Power Sources, 2019. 414: p. 158-166.

2. Zhao, L., G. Ji, and Z. Liu, Design and Experiment of Nonlinear Observer with Adaptive Gains for Battery State of Charge Estimation. Energies, 2017. 10(12).

3. Zhu, X., et al., Electrochemical impedance study of commercial LiNi0.80Co0.15Al0.05O2 electrodes as a function of state of charge and aging. Electrochimica Acta, 2018. 287: p. 10-20.

4. $\quad$ Zheng, L., et al., Incremental capacity analysis and differential voltage analysis-based state of charge and capacity estimation for lithium-ion batteries. Energy, 2018. 150(C): p. 759-769.

5. Zheng, Y., et al., Investigating the error sources of the online state of charge estimation methods for lithium-ion batteries in electric vehicles. Journal of Power Sources, 2018. 377: p. 161-188.

6. Hosseini Mehri, T., A. Ghosh, and F. Shahnia, Cooperative control of battery energy storage systems in microgrids. International Journal of Electrical Power \& Energy Systems, 2017. 87: p. 109-120.

7. Wu, J., et al., Research on Modeling and SOC Estimation of Lithium Iron Phosphate Battery at Low Temperature. Energy Procedia, 2018. 152: p. 556-561.

8. Lim, K., et al., Fading Kalman filter-based real-time state of charge estimation in LiFePO4 batterypowered electric vehicles. Applied Energy, 2016. 169: p. 40-48. 
9. Liu, G., et al., State of charge and model parameters estimation of liquid metal batteries based on adaptive unscented Kalman filter. Energy Procedia, 2019. 158: p. 4477-4482.

10. Guo, L., J. Li, and Z. Fu, Lithium-Ion Battery SOC Estimation and Hardware-in-the-Loop Simulation Based on EKF. Energy Procedia, 2019. 158: p. 2599-2604.

11. Wu, J., C. Zhang, and Z. Chen, an online method for lithium-ion battery remaining useful life estimation using importance sampling and neural networks. Applied Energy, 2016. 173: p. 134-140.

12. $\mathrm{Hu}, \mathrm{X}$., et al., State estimation for advanced battery management: Key challenges and future trends. Renewable and Sustainable Energy Reviews, 2019. 114: p. 109334.

13. Xiao Ma, D.Q., Qing Tao and Daiyin Zhu, State of Charge Estimation of a Lithium-Ion Battery Based on Adaptive Kalman Filter Method for an Equivalent Circuit Model. Applied Sciences, 2019.

14. Sharaf, A.M., et al., 12 - Electric and Hybrid Vehicle Drives and Smart Grid Interfacing, in Advances in Renewable Energies and Power Technologies, I. Yahyaoui, Editor. 2018, Elsevier. p. 413-439.

15. Xia, B., et al., Strong Tracking of a H-Infinity Filter in Lithium-Ion Battery State of Charge Estimation. Energies, 2018. 11: p. 1481.

16. Wang, D., Y. Bao, and J. Shi, Online Lithium-Ion Battery Internal Resistance Measurement Application in State-of-Charge Estimation Using the Extended Kalman Filter. Energies, 2017. 10(9): p. 1284.

17. Cano, Z.P., et al., Batteries and fuel cells for emerging electric vehicle markets. Nature Energy, 2018. 3(4): p. 279-289.

18. Chen, Z., et al., Online state of charge estimation of Li-ion battery based on an improved unscented Kalman filter approach. Applied Mathematical Modelling, 2019. 70: p. 532-544.

19. Wei He 1, M.P., David Flynn 2 and Fatemi Din Mohammadi 2*, A Physics-Based Electrochemical Model for Lithium-Ion Battery State-of-Charge Estimation Solved by an Optimised Projection-Based Method and Moving Window Filtering. energy, 2018.

20. Chen, C., et al., State-of-charge estimation of lithium-ion battery using an improved neural network model and extended Kalman filter. Journal of Cleaner Production, 2019. 234: p. 1153-1164.

21. Lai, X., et al., A comparative study of global optimization methods for parameter identification of different equivalent circuit models for Li-ion batteries. Electrochimica Acta, 2019. 295: p. 1057-1066.

22. Li, H., et al., State of charge estimation for lithium-ion battery using an electrochemical model based on electrical double layer effect. Electrochimica Acta, 2019. 326: p. 134966.

23. Xu, Y., et al., State of charge estimation for lithium-ion batteries based on adaptive dual Kalman filter. Applied Mathematical Modelling, 2020. 77: p. 1255-1272.

24. Wang, S.-L., et al., An improved packing equivalent circuit modeling method with the cell-to-cell consistency state evaluation of the internal connected lithium-ion batteries. Energy Science \& Engineering, 2019. 7(2): p. 546-556.

25. Jindong Duan 1, P.W., Wentao Ma 1*, Xinyu Qiu 2, Xuan Tian 1 and Shuai Fang 1, State of Charge Estimation of Lithium Battery Based on Improved Correntropy Extended Kalman Filter. Energies, 2020.

26. Dang, X., et al., Open-Circuit Voltage-Based State of Charge Estimation of Lithium-ion Battery Using Dual Neural Network Fusion Battery Model. Electrochimica Acta, 2016. 188: p. 356-366.

27. Zhang, W., et al., An improved adaptive estimator for state-of-charge estimation of lithium-ion batteries. Journal of Power Sources, 2018. 402: p. 422-433.

28. Zhang, Y., et al., Real-Time Study of the Disequilibrium Transfer in Vanadium Flow Batteries at Different States of Charge via Refractive Index Detection. The Journal of Physical Chemistry C, 2018. 122(50): p. 28550-28555.

29. Liu, F., J. Ma, and W. Su, Unscented Particle Filter for SOC Estimation Algorithm Based on a Dynamic Parameter Identification. Mathematical Problems in Engineering, 2019. 2019: p. 7452079.

30. Zheng, L., et al., Incremental capacity analysis and differential voltage analysis-based state of charge and capacity estimation for lithium-ion batteries. Energy, 2018. 150: p. 759-769.

31. Zheng, Y., et al., State-of-charge inconsistency estimation of lithium-ion battery pack using meandifference model and extended Kalman filter. Journal of Power Sources, 2018. 383: p. 50-58.

32. Chen, X., et al., A novel approach to reconstruct open circuit voltage for state of charge estimation of lithium-ion batteries in electric vehicles. Applied Energy, 2019. 255: p. 113758.

33. Zhang, C., et al., Online estimation of battery equivalent circuit model parameters and state of charge using decoupled least squares technique. Energy, 2018. 142: p. 678-688.

34. Shrivastava, P., et al., Overview of model-based online state-of-charge estimation using Kalman filter family for lithium-ion batteries. Renewable and Sustainable Energy Reviews, 2019. 113: p. 109233.

35. Zhang, C., et al., An Improved Model-Based Self-Adaptive Filter for Online State-of-Charge Estimation of Li-Ion Batteries. Applied Sciences, 2018. 8: p. 2084.

36. Carnevale, D., C. Possieri, and M. Sassano, State-of-charge estimation for lead-acid batteries via embeddings and observers. Control Engineering Practice, 2019. 85: p. 132-137. 
37. Xing, J. and P. Wu, State of Charge Estimation of Lithium-Ion Battery Based on Improved Adaptive Unscented Kalman Filter. Sustainability, 2021. 13(9).

38. Zhang, N., et al., A Novel Method for Estimating State-of-Charge in Power Batteries for Electric Vehicles. International Journal of Precision Engineering and Manufacturing, 2019. 20(5): p. 845-852.

39. Deng, Z., et al., Online available capacity prediction and state of charge estimation based on advanced data-driven algorithms for lithium iron phosphate battery. Energy, 2016. 112: p. 469-480.

40. Kuo Yang, Y.T.a.Z.Z., Parameter Identification and State-of-Charge Estimation for Lithium-Ion Batteries Using Separated Time Scales and Extended Kalman Filter. Energies, 2021.

41. Chao Lyu, J.L., Lulu Zhang, Michael Precht, Lixin Wang, Dafang Wang, State of charge estimation based on a thermal coupling simplified first-principles model for lithium-ion batteries. J. Energy Storage, 2019.

42. Yang, S., et al., A parameter adaptive method for state of charge estimation of lithium-ion batteries with an improved extended Kalman filter. Scientific Reports, 2021. 11(1): p. 5805.

43. Wang, H., et al., Novel estimation solution on lithium-ion battery state of charge with current-free detection algorithm. IET Circuits, Devices \& Systems, 2019. 13(2): p. 245-249.

44. Shu, X., et al., An adaptive fusion estimation algorithm for state of charge of lithium-ion batteries considering wide operating temperature and degradation. Journal of Power Sources, 2020. 462: p. 228132.

45. Zhi, L., et al., State of Charge Estimation for Li-ion Battery Based on Extended Kalman Filter. Energy Procedia, 2017. 105: p. 3515-3520.

46. Omariba, Z.B., L. Zhang, and D. Sun, Review on Health Management System for Lithium-Ion Batteries of Electric Vehicles. Electronics, 2018. 7(5).

47. Xu, W., J. Xu, and X. Yan, Lithium-ion battery state of charge and parameters joint estimation using cubature Kalman filter and particle filter. Journal of Power Electronics, 2020. 20(1): p. 292-307.

48. Lai, X., Y. Zheng, and T. Sun, A comparative study of different equivalent circuit models for estimating state-of-charge of lithium-ion batteries. Electrochimica Acta, 2018. 259: p. 566-577.

49. Homan, B., et al., A realistic model for battery state of charge prediction in energy management simulation tools. Energy, 2019. 171: p. 205-217.

50. Wei, Z., et al., Online monitoring of state of charge and capacity loss for vanadium redox flow battery based on autoregressive exogenous modeling. Journal of Power Sources, 2018. 402: p. 252-262.

51. Zhongbao Wei, J.Z., Changfu Zou, Tutti Mariana Lim, King Jet Tseng, Comparative study of methods for integrated model identification and state of charge estimation of lithium-ion battery. Journal of Power Sources, 2019. 